\title{
PERFIL SOCIOAMBIENTAL DE MORADORES QUE CULTIVAM RECURSOS VEGETAIS EM ESPAÇOS RESIDENCIAIS NO MUNICÍPIO DE GUARULHOS, SP
}

\author{
Silvia Rosana Dos Santos ${ }^{1}$
}

Maria Solange Francos ${ }^{2}$

Ana Paula do Nascimento Lamano-Ferreira ${ }^{3}$

\begin{abstract}
RESUMO
Com o crescimento dos grandes centros urbanos, a população humana altera seus hábitos em relação ao cultivo de plantas. No presente trabalho levantou-se o perfil de moradores que cultivam plantas em seus quintais e o uso das plantas cultivadas nos mesmos. Para isso, foi estudada a população dos bairros Presidente Dutra e Inocoop, no município de Guarulhos, SP. Foram realizadas 150 entrevistas e, dentre os voluntários entrevistados, a maioria foi de mulheres. Utilizou-se um roteiro de entrevistas e escolha aleatória dos domicílios pesquisados para conferir imparcialidade nos dados pesquisados. Os quintais residenciais do município de Guarulhos apresentam inúmeras finalidades além do cultivo de plantas, como espaço de lazer, trabalho doméstico, garagem de carro e abrigo para animais. Entretanto, em ambos os bairros houve a predominância de cultivo de plantas ornamentais, seguidas pelas alimentícias e pelas medicinais. Esses dados indicam preferência pelo cultivo de plantas ornamentais nos quintais da cidade de Guarulhos, SP. Uma provável explicação pode ser pela estética (ou embelezamento) oferecida pelas flores destas plantas ou o não consumo de plantas alimentícias e medicinais pelas entrevistadas.
\end{abstract}

PALAVRAS-CHAVE: Ecologia Humana. Espaços Verdes Urbanos. Sustentabilidade.

\footnotetext{
${ }^{1}$ Bióloga, Universidade Nove de Julho - UNINOVE. E-mail: asilvia.rs@gmail.com

${ }^{2}$ Professora do Departamento de Saúde da UNINOVE. E-mail:mariasolangef@yahoo.com.br

${ }^{3}$ Professora do Programa de Mestrado Profissional em Gestão Ambiental e Sustentabilidade da Universidade Nove de Julho -GeAS/UNINOVE. E-mail: apbnasci@yahoo.com.br
} 


\title{
SOCIAL AND ENVIRONMENTAL PROFILE OF RESIDENTS THAT CULTIVATE PLANTS IN RESIDENTIAL SPACES IN GUARULHOS, SP
}

\begin{abstract}
With the growth of large urban centers, the human population alters their habits regarding the cultivation of plants. In the present work raised the profile of residents who grow plants in their backyards and the use of cultivated plants in the same. Was also investigated using the human population carries these resources. For this, the population of the districts Presidente Dutra and INOCOOP, in Guarulhos, SP, was studied. 150 interviews were conducted in neighborhoods Presidente Dutra and INOCOOP. Among the volunteers interviewed, the majority were women. We used a set of interviews and random selection of households surveyed to check impartiality in research data. Residential spaces in the municipality of Guarulhos have several purposes beyond the cultivation of plants, a place of leisure, housework, car garage and animal shelter. However, in both areas predominated for cultivating ornamental plants and they represented, followed by the food and medicinal. These data indicate preference for the cultivation of ornamental plants in the backyards of the city of Guarulhos, SP. A likely explanation may be the aesthetic (or embellishment) offered by the flowers of these plants or non-consumption of food and medicinal plants by the interviewees.
\end{abstract}

KEYWORDS: Human Ecology. Urban Green Spaces. Sustainability

\section{PERFIL AMBIENTAL DE LOS RESIDENTES QUE UTILIZAN LOS RECURSOS VEGETALES QUINTALES EN GUARULHOS, SP}

\section{RESUMEN}

Con el crecimiento de los grandes centros urbanos, la población humana altera sus hábitos en relación con el cultivo de plantas. En el presente trabajo elevado el perfil de los residentes que crecen las plantas en sus patios traseros y el uso de las plantas cultivadas en el mismo. También se investigó el uso de la población humana lleva a estos recursos. Para ello, la población de los distritos Presidente Dutra y INOCOOP, en Guarulhos, se estudió SP. 150 entrevistas se llevaron a cabo en los barrios Presidente Dutra y INOCOOP. Entre los voluntarios entrevistados, la mayoría eran mujeres. Utilizamos una serie de entrevistas y selección aleatoria de los hogares encuestados para comprobar la imparcialidad en los datos de investigación. Patios residenciales en el municipio de Guarulhos tienen varios propósitos más allá del cultivo de las plantas, un lugar de ocio, tareas del hogar, garaje y refugio de animales. Sin embargo, en ambas áreas predominaron para el cultivo de plantas ornamentales y que representaban, seguido de la comida y medicinal. Estos datos indican preferencia por el cultivo de plantas ornamentales en los patios de la ciudad de Guarulhos, SP. Una explicación probable puede ser la estética (o adorno) ofrecido por las flores de estas plantas o no el consumo de alimentos y plantas medicinales por parte de los entrevistados.

PALABRAS-CLAVE: Ecología Humana. Urbanos Espacios Verdes. Sostenibilidad. 


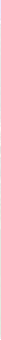

\section{INTRODUÇÃO}

Os quintais residenciais representam uma das formas mais antigas de manejo da terra (AMARAL; GUARIM NETO, 2008). Esses sistemas constituem uma combinação de árvores, arbustos, herbáceas, algumas vezes associadas a pequenos animais domésticos.

No Brasil, quintal é o termo mais comum para designar o espaço no entorno da residência, no qual podem ser cultivadas espécies que fornecem parte da dieta nutricional da família (NASCIMENTO et al., 2005), como também conservação da biodiversidade (ALTHAUS-OTTMANN et al., 2010).

A prática de cultivo de plantas nos quintais contribui para melhorar aspectos importantes da vida em centros urbanos, no que se refere à segurança alimentar, pois permite uma diversificação na dieta (NASCIMENTO et al., 2005). A inclusão de hortaliças na dieta alimentar apresenta vantagens nutricionais, pois são fontes de vitaminas e sais minerais, além de trazer outros benefícios como favorecer melhor funcionamento do intestino devido à presença de fibras e celulose, trazendo impactos positivos para a saúde (NOBREGA, 1998 apud. HORN, 2006). De acordo com Amaral e Guarim Neto (2008), uma dieta rica em hortaliças reduz os riscos de desenvolvimento de doenças cardiovasculares, câncer e outras doenças crônicas. A agricultura na cidade tem interfaces importantes com diversos aspectos do ecossistema urbano, que vão da produção de alimentos ao resgate da cultura, em relação ao cuidado com o meio ambiente e com as plantas, e podem propiciar novas sociabilidades (MONTEIRO, 2003). Desse modo, esses espaços verdes residenciais podem contribuir para a manutenção da diversidade biológica em todas as suas modalidades rurais, urbanas e periurbanas (LACERDA, 2008).

A relação entre populações humanas e recursos vegetais tem sido abordada ao longo de toda história do conhecimento científico. O termo etnobotânica refere-se ao estudo desta relação e começou a ser utilizado há pouco mais de um século, sendo originalmente o estudo das plantas utilizadas por população tradicional. Esta área do conhecimento tem sido definida como o estudo das interelações entre seres humanos e plantas (OLIVEIRA et al., 2009). A investigação etnobotânica possibilita comparar diferentes áreas de estudo, facilitando a descoberta de distintas maneiras 
de uso de um mesmo recurso vegetal. Além disso, auxilia na compreensão do etnoconhecimento de uma população, contribuindo para a conservação desses recursos (MIRANDA; HANAZAKI, 2008; DIEGUES, 2010). Essa noção torna a etnobotânica algo próprio de uma cultura ou de uma sociedade (OLIVEIRA et al., 2009).

Com o crescente processo de urbanização, exploração de recursos naturais e redução de áreas verdes (SILVA; VARGAS, 2010), as cidades estão se tornando importante foco de estudo. De acordo com os autores, áreas verdes cumprem funções importantes como a de produzir oxigênio, modificar o microclima, absorver poluentes, além de satisfazer questões estéticas. Portanto, a importância dos espaços residenciais para cultivo de recursos vegetais torna-se relevante, podendo contribuir também com a manutenção do ecossistema urbano. Cabe ressaltar que usos de plantas para fins alimentícios (SIVIERO et al., 2011) inclui o princípio de sustentabilidade e constitui-se numa estratégia de promoção de saúde, manutenção da biodiversidade e transmissão de conhecimento para as gerações mais jovens (BALDAUF et al., 2009).

Diante desta constatação, torna-se relevante conhecer as relações humanas com os recursos vegetais em grandes metrópoles. Assim, o presente trabalho se propôs a responder as seguintes perguntas: i) Há ocorrência de quintais na cidade de Guarulhos, SP?; ii) Qual o perfil socioambiental do morador que cultiva recursos vegetais?; iii) Para quais finalidades as plantas são cultivadas nos quintais?

\section{DESENVOLVIMENTO}

\section{Metodologia}

\section{Coleta de dados}

O trabalho foi desenvolvido com os moradores dos bairros Presidente Dutra (PD) e Inoocop (IN) que concordaram em participar da presente pesquisa. Participaram preferencialmente mulheres, através de entrevistas realizadas nos domicílios, após sorteio de cinco ruas por bairro e as residências destas ruas visitadas. De acordo com a literatura (NASCIMENTO et al., 2006; FREITAS et al., 
2012), a manutenção dos quintais e os vegetais neles cultivados, ou em qualquer outra área da residência, são de responsabilidade das mulheres. Elas decidem o que, como, onde e quando serão plantados ou não (SHIVA; DALKEMAN, 1994).

Cada morador que participou deste estudo recebeu um Termo de Consentimento Livre e Esclarecido sobre a pesquisa, o qual foi assinado, permitindo assim o uso das informações geradas nas entrevistas, bem como o uso das fotografias tiradas dos quintais.

O instrumento utilizado foi um roteiro semi estruturado com perguntas abertas e fechadas (MARCONI; LAKATOS, 2002), sendo incorporadas informações adicionais consideradas relevantes. Foram coletadas informações sobre a presença de quintal na residência, hábitos de cultivo e utilização das plantas e perfil sócio ambiental (idade, nível de escolaridade, classe econômica, religiosidade, tempo de residência no município de Guarulhos). Os dados foram analisados de forma quantitativa.

\section{Análise dos dados}

Foi verificada a finalidade das plantas cultivadas nos quintais urbanos $(1=$ plantas alimentícias; 2 = plantas medicinais e 3= plantas ornamentais), como também sua utilização para fins alimentícios e/ou medicinais. Para as plantas medicinais foram obtidos dados sobre o órgão da planta utilizado e suas indicações de uso.

\section{Resultados e Discussão}

$\mathrm{Na}$ Tabela 1 observa-se que a maioria dos entrevistados possui mais de 50 anos (54,9\%: PD; 64,6\% IN), seguido pela faixa etária de 41 a 50 anos (23,5\%); 61 anos ou mais (22,5\%); 20 a 30 anos (10,8\%) e 31 a 40 anos (10,8\%) no bairro PD. Já no bairro IN 25\% dos entrevistados estão na faixa etária 61 ou mais, seguido por $16,7 \%$ entre 41 e 50 anos, 12,5\% entre 31 e 40 anos e 6,3\% de 20 a 30 anos. Dessa forma, pode-se dizer que a faixa etária mais presente nos domicílios ou que tinha mais disponibilidade em responder a presente pesquisa corresponde a idades entre 51 e 60 anos e os que menos respondem estão na faixa de 20 a 30 anos. 
Quanto ao nível de escolaridade, observa-se uma diferença em relação ao tempo de estudo nos bairros pesquisados, sendo que o bairro PD concentra maior parte dos entrevistados nas faixas de 0 a 3 anos de estudo (52\%). Nesta mesma faixa de escolaridade, o bairro Inocoop apresenta o índice de (29,2\%) dos entrevistados. No entanto, a maioria dos participantes da pesquisa deste mesmo bairro concluiu o ensino médio (39,6\%), enquanto no bairro PD, $12,7 \%$ dos entrevistados concluíram o ensino médio. Esses resultados mostram que no bairro IN $48 \%$ possuem Ensino Médio completo enquanto no bairro PD a maioria (52\%) dos entrevistados estudou até 3 anos.

A maior parte da população pesquisada possui companheiro (a), tanto no bairro PD (67,6\%) como no bairro IN (64,6\%), enquanto no bairro PD o número de filhos é menor, apresentando dois filhos por casal (32,4\%). Entretanto, no bairro IN o número de filhos por casal sofre um acréscimo e grande parte dos casais tem três filhos ou mais $(47,9 \%)$.

Em relação às crenças religiosas da população estudada, verificou-se diversidade. Em ambos os bairros verifica-se maior número entre os que declararam católicos (PD: 54,8\%; IN 54,1\%), seguido por evangélicos (PD: 30,9\%; IN: 29,1\%) e espíritas (PD: 4,8\%; IN: 10,4\%). Foram identificadas outras crenças religiosas como budismo no bairro PD (2,3\%), não verificada no bairro IN e messiânicos no IN $(4,1 \%)$, não verificado no bairro PD. Em ambos os bairros houve casos em que os entrevistados declararam não ter religião PD $(7,1 \%)$ e IN (2\%), e ainda no bairro IN, houve caso onde os entrevistados declararam-se espiritualistas (2\%). O presente trabalho compartilha semelhança com o estudo realizado por Freitas (2009) no que diz respeito ao maior número de entrevistados pertencentes às religiões católica e evangélica. No citado estudo, realizado no município de São Miguel, foram encontrados $95 \%$ de católicos e $5 \%$ de evangélicos, mas há que considerar a discrepância entre os índices, pois o número de evangélicos é menor, enquanto na presente pesquisa há maior equilíbrio entre a quantidade de fiéis que se declaram católicos e evangélicos.

Em relação à presença de quintais, em ambos os bairros pesquisados há grande ocorrência (PD: 92,2\%; IN: 79,2\%). De acordo com os entrevistados, tanto no bairro PD $(96,1 \%)$ quanto no IN $(97,9 \%)$ encontram-se recursos vegetais em 
proporções semelhantes em relação à presença de plantas nas residências. Pôdese verificar que os quintais também são utilizados para outras funções como: local de lazer, de realização de trabalho doméstico, garagem, entre outras, mas vai além das funcionalidades domésticas, pois conservam em seu interior estoque de recursos vegetais (Tabela 1 ).

Tabela 1. Perfil socioambiental da população dos bairros Presidente Dutra $(n=102)$ e Inocoop $(n=48)$ domunicípio de Guarulhos, SP, no ano de 2012-2013.

\begin{tabular}{|c|c|c|c|c|}
\hline \multirow[t]{2}{*}{ VARIÁVEIS } & \multicolumn{2}{|c|}{$\begin{array}{l}\text { PRESIDENTE DUTRA } \\
\qquad \mathrm{N}=102\end{array}$} & \multicolumn{2}{|c|}{$\begin{array}{c}\text { INOCOOP } \\
N=48\end{array}$} \\
\hline & $\mathrm{N}$ & $\%$ & $\mathrm{~N}$ & $\%$ \\
\hline \multicolumn{5}{|l|}{ FAIXA ETÁRIA } \\
\hline 20 a 30 anos & 11 & 10,8 & 03 & 6,3 \\
\hline 31 a 40 anos & 11 & 10,8 & 06 & 12,5 \\
\hline 41 a 50 anos & 24 & 23,5 & 08 & 16,7 \\
\hline 51 a 60 anos & 33 & 32,4 & 19 & 39,6 \\
\hline 61 ou mais & 23 & 22,5 & 12 & 25 \\
\hline \multicolumn{5}{|l|}{ NIVEL DE ESCOLARIDADE } \\
\hline 0 a 3 anos & 53 & 52,0 & 14 & 29,2 \\
\hline 4 a 7 anos & 18 & 17,6 & 07 & 14,6 \\
\hline 8 a 11 anos & 13 & 12,7 & 04 & 8,3 \\
\hline Ensino Médio Completo & 13 & 12,7 & 19 & 39,6 \\
\hline Ensino Superior Completo & 05 & 4,9 & 04 & 8,3 \\
\hline \multicolumn{5}{|l|}{ SITUAÇÃO CONJUGAL } \\
\hline Com companheiro (a) & 69 & 67,6 & 31 & 64,6 \\
\hline Sem companheiro (a) & 33 & 32,4 & 17 & 35,4 \\
\hline \multicolumn{5}{|l|}{ CLASSE ECONÔMICA } \\
\hline A & - & - & 03 & 6,3 \\
\hline B & 10 & 9,8 & 40 & 83,3 \\
\hline C & 58 & 56,9 & 05 & 10,4 \\
\hline D & 22 & 21,6 & 00 & 0 \\
\hline \multicolumn{5}{|l|}{ NÚMERO DE FILHOS } \\
\hline Um & 21 & 21,6 & 08 & 16,7 \\
\hline Dois & 33 & 32,4 & 12 & 25 \\
\hline Três ou mais & 29 & 28,4 & 23 & 47,9 \\
\hline Não possui & 19 & 18,6 & 05 & 10,4 \\
\hline \multicolumn{5}{|l|}{ RELIGIÁO } \\
\hline Budismo & 01 & 2,3 & 0 & 0 \\
\hline Católica & 23 & 54,8 & 26 & 54,1 \\
\hline Espírita & 02 & 4,76 & 05 & 10,4 \\
\hline Espiritualista & 0 & 0 & 01 & 2 \\
\hline Evangélico & 13 & 30,9 & 14 & 29,1 \\
\hline Messiânico & 0 & 0 & 02 & 4,1 \\
\hline Sem religião & 03 & 4,6 & 01 & 2 \\
\hline \multicolumn{5}{|l|}{ TEMPO DE RESIDÊNCIA } \\
\hline 0 a 3 & 06 & 6 & 01 & 2 \\
\hline 4 a 6 & 5 & 5 & 04 & 8,3 \\
\hline 7 a 10 & 4 & 4 & 03 & 6,2 \\
\hline 11 a 15 & 7 & 6,9 & 02 & 4,7 \\
\hline 16 a 20 & 20 & 19,7 & 04 & 8,3 \\
\hline
\end{tabular}




\begin{tabular}{lcccc}
+ de 20 & 60 & 59 & 34 & 70,9 \\
$\begin{array}{l}\text { QUINTAL } \\
\text { Presença }\end{array}$ & 94 & 92,2 & 38 & 79,2 \\
Recursos Vegetais & 98 & 96,1 & 47 & 97,9 \\
\hline
\end{tabular}

Verificou-se que nos quintais residenciais urbanos do município de Guarulhos, SP, tanto no bairro Presidente Dutra quanto no bairro Inocoop, as plantas mais cultivadas são para fins ornamentais (PD: 63\% e IN: 68,9\%), como mostra a Figura 1. Em seguida, no bairro PD observa-se que 22,3\% dos moradores entrevistados cultivam plantas alimentícias seguida de $14,7 \%$ que cultivam plantas medicinais. Já no bairro IN, 18,4\% cultivam plantas medicinais, seguido por $12,6 \%$ dos entrevistados que cultivam plantas alimentícias. Era esperado que, em uma metrópole como Guarulhos, as plantas mais cultivadas fossem ornamentais, como encontrado em outros trabalhos realizados em capitais como Curitiba, PR (ALTHAUS-OTMMAN et al., 2010) e Porto Alegre, RS (VENDRUSCOLO; MENTZ, 2006; BALDAUF et al., 2009). Cabe destacar que são escassos os trabalhos de etnobotânica que envolvam as diferentes finalidades de cultivo, pois os trabalhos mais encontrados são com plantas medicinais seguida de alimentícias.

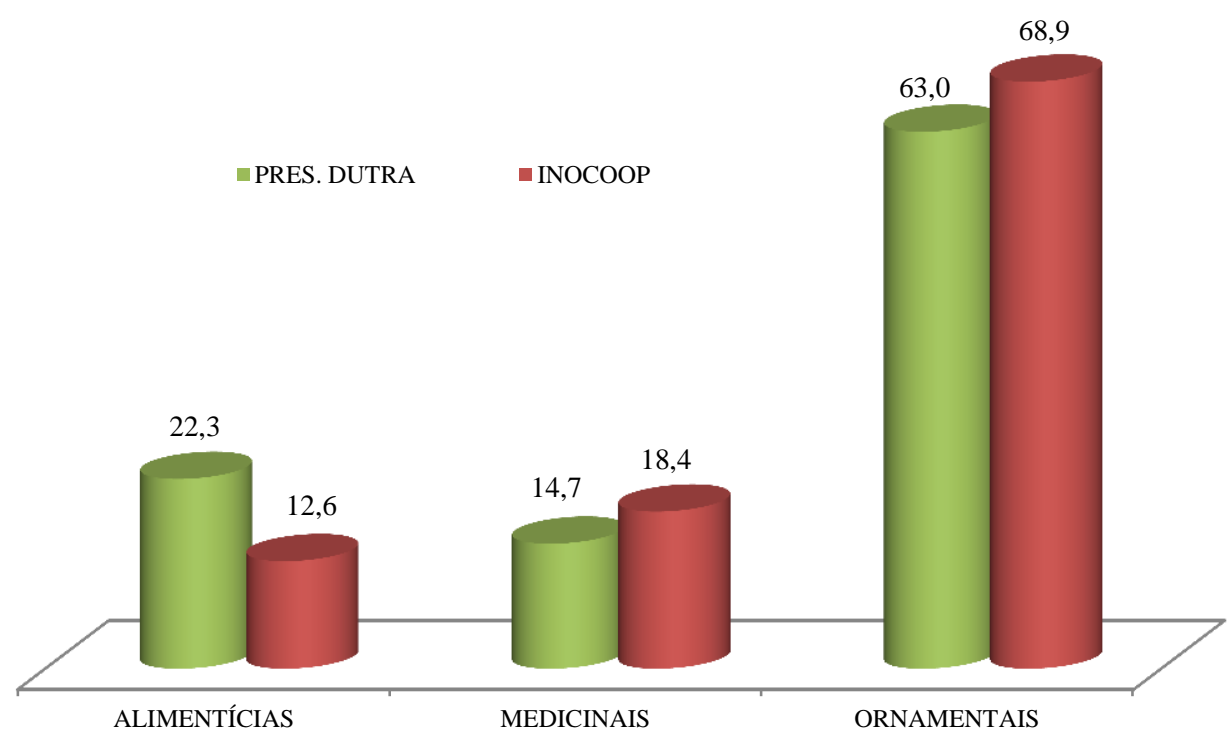

Figura 1. Finalidade de plantas cultivadas nos espaços residenciais dos bairros Presidente Dutra e Inocoop no município de Guarulhos, SP. 
Foram relatadas 41 categorias de plantas alimentícias entre os informantes do município de Guarulhos, SP. Entretanto as plantas encontradas nos espaços residenciais de Guarulhos ainda estão sendo identificadas e será foco do próximo trabalho da equipe.

Em relação às plantas medicinais, foram encontradas 21 plantas. Já no bairro IN foram encontradas 15 plantas para uso medicinal. A folha é a parte da planta mais utilizada pela população estudada no bairro PD, que possui plantas medicinais chegando a $100 \%$ dos entrevistados, entretanto outros órgãos das plantas são utilizados, tais como caule $(9 \%)$, raiz $(4,5 \%)$, casca $(2,9 \%)$, semente $(2,9 \%)$ e flor $(1 \%)$.

No bairro IN o uso da folha ocorre em $95,7 \%$ dos casos, seguida do fruto $4,3 \%$ e caule $4,3 \%$; o modo de preparo mais comum, com finalidade medicinal, é o chá, em ambos os bairros do presente estudo. Porém, as mesmas plantas aplicamse a vários tratamentos de saúde: a hortelã é usada para doenças como a tosse, gripe, dor de cabeça, problemas no trato gastrintestinal, dor de barriga, flatulência, como vermífugo e calmante; o boldo é usado para problemas de saúde associados ao trato gastrintestinal, como também problemas no fígado. Outra forma de uso é para picada de insetos e queimadura; o alecrim é usado para problemas psicoemocionais como nervosismo, cardiopatias, pressão alta e doenças respiratórias, como resfriado; a babosa é usada para queimaduras, como cicatrizante, para tratamento de doenças do trato gastrintestinal, inflamações e preventivo para câncer.

\section{CONCLUSÃO}

O perfil socioambiental dos moradores que cultivam plantas em espaços residenciais no município de Guarulhos mostrou que estes possuem, em sua maioria, mais de 50 anos de idade, até 3 anos de estudo ou 12 anos completos (Ensino Médio), mulher com companheiro e pelo menos 1 filho. Além disso, pertencem às classes $B(I N)$ ou $C$ (PD).

Constatou-se grande ocorrência de quintais residenciais nos bairros Presidente Dutra e Inocoop, do município de Guarulhos, SP, como também cultivo 


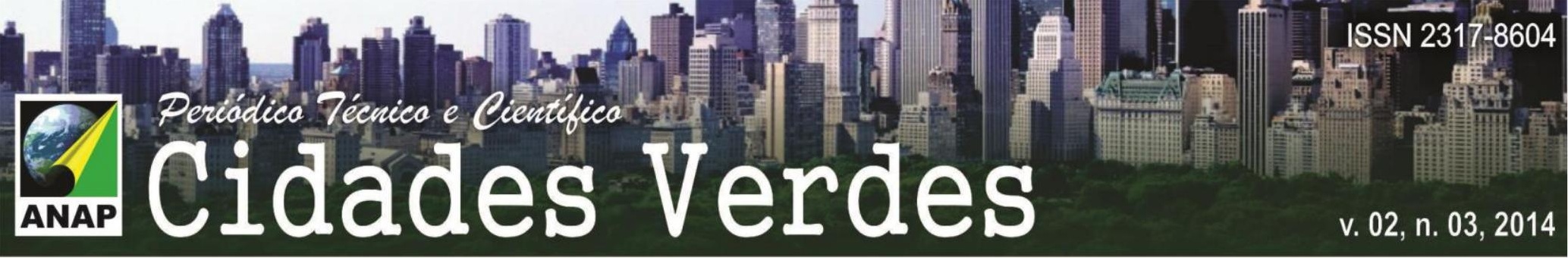

de plantas na maioria destes. As plantas mais cultivadas nestes quintais são para fins ornamentais, seguidas pelas alimentícias e, por último, pelas medicinais no bairro PD, apesar do IN também apresentar maior predominância das plantas ornamentais, seguidas por plantas medicinais e alimentícias.

Agradecimento(s): A Universidade Nove de Julho pelo incentivo e apoio a pesquisa e ao Prof. Maurício Lamano Ferreira pelas discussões durante a execução e identificação das plantas.

\section{REFERÊNCIAS}

ALMADA, E. D.; SILVA, V. A.; ALMEIDA, A. L.; ALBUQUERQUE, U. P. Sociobiodiversidade Urbana: por uma etnoecologia das cidades. In:. Etnobiologia e Etnoecologia: Pessoas \& Natureza na América Latina. Recife: Nuppea, 2010. Cap. 1, p. 39-63.

ALTHAUS-OTTMANN, M. M.; CRUZ, M. J. R.; FONTE, N. N. Diversidade e uso de plantas cultivadas nos quintais do Bairro Fanny, Curitiba, Paraná, Brasil. Revista Brasileira de Biociências. Porto Alegre, 9(1): 39-49, 2010.

AMARAL, C. N.; GUARIM NETO, G. Os quintais como espaço de conservação e cultivo de alimentos: um estudo na cidade de Rosário Oeste (Mato Grosso, Brasil). Bol. Mus. Emílio Goeldi. Ciências Humanas, Belém, 3(3): 329-341, 2008.

BALDAUF, C.; KUBO, R. R.; SILVA, F.; IRGANG, B. E. "Ferveu, queimou o ser da erva": conhecimento de especialistas locais sobre plantas medicinais na região Sul do Brasil. Revista Brasileira de Plantas Medicinais. Botucatu, 11(3): 282-291, 2009.

DIEGUES, A. C. (org.). Etnoconservação: novos rumos para proteção da natureza nos trópicos. São Paulo: HUCITEC, 2000.

FREITAS, A. V. L.; COELHO, M. F. B.; MAIA, S. S. S. S; AZEVEDO, R. A. B. Plantas medicinais: um estudo etnobotânico nos quintais do Sítio Cruz, São Miguel, Rio Grande do Norte, Brasil. Revista Brasileira de Biociências. Porto Alegre 1(1): 48-59,2012.

HORN, M. A. Implantação de Uma Horta Educativa na Escola Municipal "Vila Irene": O Cultivo de Hortaliças como Instrumento de Ensino e Promoção de Saúde. Monografia, Universidade de Mato Grosso, Cáceres, 2006.

LACERDA,V. D. Quintais do Sertão do Ribeirão: Agrobiodiversidade Sob Um Enfoque Etnobotânico. Monografia de Ciências Biológicas, Centro de Ciências Biológicas da Universidade Federal de Santa Catarina, Florianópolis, 2008.

MARCONI, M. A.; LAKATOS, E. M. Técnicas de Pesquisa: planejamento e execução de pesquisas, amostragens e técnicas de pesquisas, elaboração, análise e interpretação de dados. São Paulo: Atlas, 2002.

MIRANDA, T. M.; HANAZAKI, N. Conhecimento e uso de recursos vegetais de restinga por comunidades das ilhas do Cardoso (SP) e de Santa Catarina (SC), Brasil. Acta Bot. Bras. 22(1): 203$215,2008$. 
MONTEIRO, D.; MENDONÇA; M. M. Quintais na Cidade: a experiência de moradores da periferia do Rio de Janeiro, 2003. Disponível em: www.agricultures.nrtwork.org/magazines/brasil.

NASCIMENTO, A. P. B., ALVES, M. C., MOLINA, S. M. G. Condições Sócio-ambientais de Migrantes Mineiros em Piracicaba, SP, Brasil. Interciências, (30): 555- 559, 2005.

NASCIMENTO, A. P. B.; ALVES, M. C.; MOLINA, S. M. G. Quintais Domésticos e sua relação com o Estado Nutricional de crianças rurais, urbanas e migrantes. Multiciência. Campinas. v.5, 2005.

NASCIMENTO, A. P. B.; SILVA, M. R.; GARAVELLO, M. E. P. E.; MOLINA, S. M. G. Quintais domésticos: conhecimento,tradição e utilidades no município de Piracicaba,SP In: Environmental and Health Wold Congress,Santos.Natural Resources for the Health of Future Generations. p.462-464, 2006.

OLIVEIRA, F. C.,ULYSSES, P. A., KRUEL,F., HANAZAKI,N. Avanços nas Pesquisas Etnobotânicas no Brasil, Acta Bot.Bras. São Paulo, 23(2), 2009.

RÉGIS, M. M; LAMANO-FERREIRA, A. P. N. Plantas Cultivadas Em Quintais Urbanos, Zona Leste Do Município De São Paulo, SP. XI CONGRESSO DE ECOLOGIA DO BRASIL, Porto Seguro, 2013.

SHIVA, V.; DANKELMAN, I. As mulheres e a diversidade biológica: lições do Himalaia indiano In: GAIFAMI, A. (org). Cultivando a diversidade: recursos genéticos e segurança alimentar local. Rio de Janeiro: AS-PTA, p. 35-40, 1994.

SIVIERO, A.; DELUNARDO, T. A.; HAVERROTH, M.; OLIVEIRA, L. C.; MENDONÇA, A. M. S. Cultivo de Espécies Alimentares em Quintais Urbanos de Rio Branco, Acre, Brasil. Acta Bot. Bras. 25(3): 549-556, 2011.

VENDRUSCOLO, G. S., MENTZ, L. A. Levantamento etnobotânico das plantas utilizadas como medicinais por moradores do bairro Ponta Grossa, Porto Alegre, Rio Grande do Sul, Brasil. Iheringia Sér. Bot., Porto Alegre, 61(1-2): 83-103, jan./dez. 2006. 